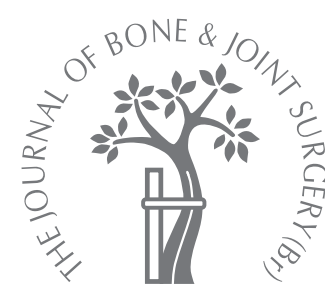

\title{
Articular cartilage restoration in load-bearing osteochondral defects by implantation of autologous chondrocyte-fibrin constructs
}

\author{
AN EXPERIMENTAL STUDY IN SHEEP
}
S. Munirah,
O. C. Samsudin,
H. C. Chen,
S. H. Sharifah
Salmah,
B. S. Aminuddin,
B. H. I. Ruszymah

From Universiti

Kebangsaan

Malaysia, Kuala

Lumpur, Malaysia

\begin{abstract}
Ovine articular chondrocytes were isolated from cartilage biopsy and culture expanded in vitro. Approximately 30 million cells per $\mathrm{ml}$ of cultured chondrocytes were incorporated with autologous plasma-derived fibrin to form a three-dimensional construct. Fullthickness punch hole defects were created in the lateral and medial femoral condyles. The defects were implanted with either an autologous 'chondrocyte-fibrin' construct (ACFC), autologous chondrocytes (ACI) or fibrin blanks (AF) as controls. Animals were killed after 12 weeks. The gross appearance of the treated defects was inspected and photographed. The repaired tissues were studied histologically and by scanning electron microscopy analysis.

All defects were assessed using the International Cartilage Repair Society (ICRS) classification. Those treated with ACFC, ACI and AF exhibited median scores which correspond to a nearly-normal appearance. On the basis of the modified O'Driscoll histological scoring scale, ACFC implantation significantly enhanced cartilage repair compared to $\mathrm{ACl}$ and $\mathrm{AF}$. Using scanning electron microscopy, $\mathrm{ACFC}$ and $\mathrm{ACl}$ showed characteristic organisation of chondrocytes and matrices, which were relatively similar to the surrounding adjacent cartilage.

Implantation of ACFC resulted in superior hyaline-like cartilage regeneration when compared with $\mathrm{ACl}$. If this result is applicable to humans, a better outcome would be obtained than by using conventional $\mathrm{ACI}$.
\end{abstract}

Articular cartilage has limited healing potential. ${ }^{1}$ Cartilage injuries, either partial thickness or full thickness with osteochondral pathology, can lead to premature arthritis ${ }^{2}$ and produce deleterious effects in the knee. ${ }^{3}$ In the hope of treating these injuries, numerous studies have been carried out in laboratory animals focusing mainly on transplantation of the reconstructed hybrid 'scaffold-cells' construct. These experiments have required the incorporation of cultured cells into polymer scaffolds, such as medical-grade polycaprolactone (mPCL), ${ }^{4}$ bilayer type I/III collagen membrane, ${ }^{5}$ atelocollagen sponge/poly-1-lactic acid (PLLA) mesh composite, ${ }^{6}$ poly (d,1)-lactide-coglycolide (PLGA)-based biomaterials, ${ }^{7}$ and fibrin glue. ${ }^{8}$ Alongside the current advancement of tissue engineering and regenerative technology, attempts to incorporate cultured cells into suitable biodegradable scaffolds are essential to improve the biological and structural properties of the engineered construct.

Several researchers have studied the potential of osteochondral restoration, in either the femoropatellar groove, ${ }^{6,9,10}$ the trochlear groove $^{11}$ or the femoral condyles ${ }^{5}$ of the rabbit.
Most have reported good results. Theoretically the femoropatellar and trochlear groove defect models are not representative of the situation faced in most patients. Anatomically, these two sites bear less weight and the lining of articular cartilage is thinner than in high weight-bearing areas. ${ }^{4}$ Therefore, we decided to study the restoration of osteochondral defects within high weight-bearing areas in a sheep model in an attempt to better simulate the clinical situation. We initiated these pre-clinical experiments by maintaining the animal model up to three months post-transplantation, based on a previous study ${ }^{12}$ in which it was reported that there were no significant differences in cartilage healing at three months or six months in an animal model.

Autologous chondrocyte implantation (ACI) was first described by Brittberg et $\mathrm{al}^{13}$ and subsequently reviewed in $2001 .^{14}$ It has been widely used to promote the healing of symptomatic articular defects in patients with success rates of about $90 \% .{ }^{15}$ However, there have been reports of failure. ${ }^{16,17}$ The implantation of a tissue-engineered cartilage construct was one of the early treatments proposed to restore chondral defects. ${ }^{10,18}$ 
We have carried out several in vitro studies to engineer 'chondrocyte-fibrin' constructs using different sources of chondrocytes, ${ }^{19}$ specifically human articular chondrocytes, ${ }^{20-22}$ nasal septum chondrocytes ${ }^{23-25}$ and auricular chondrocytes. ${ }^{26-28}$ Although fibrin is unstable and quickly disintegrates, its use as a natural polymer scaffold for tissue engineering is increasing. ${ }^{29}$ Fibrin is easily polymerised and moulded from its basic constituents. ${ }^{30}$ Moreover, fibrin is non-cytotoxic, biocompatible and biodegradable. ${ }^{31,32} \mathrm{~A}$ study of in vivo auricular cartilage repair in rabbits using autologous fibrin and chondrocytes showed encouraging results. ${ }^{33}$ A slow degradation rate was achieved by stabilising the autologous 'fibrin-chondrocyte' construct using anti-fibrinolytic agents such as aprotinin and tranexamic acid. ${ }^{33}$ However, some studies suggest that fibrin glue is not an ideal carrier to restore large articular cartilage defects in weight-bearing areas as fibrin is rapidly degraded ${ }^{4}$ and actively disintegrated. ${ }^{8}$ The latter leads to the repaired tissues having insufficient potential to resist mechanical forces. To date, we have successfully engineered our own laboratory-grown chondrocyte-fibrin construct ${ }^{34-36}$ and have investigated an autologous chondrocyte-fibrin construct (ACFC) in a sheep model. ${ }^{37,38}$

In this study, we aimed to evaluate the potential of the novel ACFC implantation technique to obtain early hyalinelike cartilage regeneration in an osteochondral defect. We evaluated the gross appearance of the treated defects, histological changes and integration of the implanted ACFC with the adjacent tissues. We assessed the performance of ACFC compared with conventional ACI and autologous fibrin (AF) blank. We hypothesised that ACFC would promote early chondrogenesis and induce hyaline-like cartilage regeneration at 12 weeks post-operation.

\section{Materials and Methods}

With the approval of the Animal Ethics Committee ${ }^{39}$ and the Research Committee of the Medical Faculty, National University of Malaysia, 12 healthy six-month-old male sheep with a mean weight of $20 \mathrm{~kg}$ (15 to 25) were used in the study. Two arthrotomy steps were required, first to harvest cartilage biopsy for in vitro expansion of chondrocytes, and secondly to implant the ACFC or ACI.

All operations were performed under general anaesthesia. After premedication with an intramuscular injection of $0.1 \mathrm{mg} / \mathrm{kg}$ xylazine (Xylaxil-20, Troy Laboratories Pty Limited, Smithfield, Australia) and $2.0 \mathrm{mg} / \mathrm{kg}$ of tramadol (Domadol, Unichem Laboratories Ltd, Mumbai, India), intravenous (IV) access was established at the elbow. Sheep were pre-oxygenated and induced with $5.0 \mathrm{mg} / \mathrm{kg}$ to $10.0 \mathrm{mg} / \mathrm{kg}$ IV ketamine (Bioketan, Vetoquinolo Biowet, Gorzów, Poland). The animals were intubated and maintained on halothane with $100 \%$ oxygen. Ventilation was controlled to maintain end-tidal carbon dioxide of less than $45 \mathrm{mmHg}$. A bolus of normal saline was administered at $20 \mathrm{ml} / \mathrm{kg} \mathrm{IV}$, immediately after induction, followed by continuous infusion at $5 \mathrm{ml} / \mathrm{kg} / \mathrm{h}$ to $10 \mathrm{ml} / \mathrm{kg} / \mathrm{h}$. Intra-operative monitoring consisted of electrocardiography, pulse oximetry, non-invasive blood pressure and end-tidal carbon dioxide measurements. All animals received intramuscular antibiotics, $1.0 \mathrm{ml} / 10 \mathrm{~kg}$ amoxicillin (Betamox LA injection, Norbrook Laboratories Limited, Carlisle, United Kingdom) for peri-operative infection prophylaxis and every other day for one week afterwards. Intramuscular meloxicam (Metacam, Boehringer Ingelheim Pty Limited, Auckland, New Zealand) $0.2 \mathrm{mg} / \mathrm{kg}$ was used for three days for post-operative pain relief.

Isolation and cultivation of articular chondrocytes. The right stifle joint region was shaved and disinfected. A medial incision of about $5.0 \mathrm{~cm}$ long was made, followed by a medial parapatellar incision of the capsule. The patella was dislocated laterally and parts of Hoffa's fat pad were resected. A full-thickness punch biopsy was harvested at the trochlear groove using a cylindrical hollow punch with an inner diameter of $5.0 \mathrm{~mm}$ and a wall thickness of $0.5 \mathrm{~mm}$. The cartilage layer was separated from the underlying bone and was used for chondrocyte isolation, in vitro cultivation, and preparation of the ACFC.

The cartilage was rinsed with phosphate-buffered saline (PBS; Gibco/Invitrogen, Grand Island, New York) and diced into approximately $1.0 \mathrm{~mm}^{3}$ segments. Chondrocytes were isolated enzymatically using $0.6 \%(\mathrm{w} / \mathrm{v})$ collagenase type II (Gibco/Invitrogen) in an orbital incubator (Stuart Scientific, Redhill, United Kingdom) at $37^{\circ} \mathrm{C}$ for six hours. Chondrocytes were strained from undigested tissue debris and cell clumps using a $40.0 \mu \mathrm{m}$ polyethersulfone mesh (Falcon, Franklin Lakes, New Jersey). The isolated chondrocytes were washed twice with PBS to remove residual collagenase. After final centrifugation at $6000 \mathrm{rpm}$ for $5 \mathrm{~min}$ at $37^{\circ} \mathrm{C}$ (Jouan centrifuge, Duguay Trouin, St Herblain, France) the cell pellet was resuspended in PBS for total cell count using a haemocytometer (Weber Scientific International Ltd, Middlesex, United Kingdom). Cell viability was determined using $0.4 \%$ trypan blue dye exclusion test (Gibco/Invitrogen). Isolated chondrocytes were seeded in six-well plates (Falcon) with an initial seeding of $5000 \mathrm{cells} / \mathrm{cm}^{2}$ in F12:DMEM (1:1) supplemented with $10 \%$ (v/v) Lamb Serum (Gibco/Invitrogen), antibiotic and antimycotic (Gibco/Invitrogen), $200 \mathrm{mM}$ 1-glutamine (Gibco/Invitrogen), $50 \mu \mathrm{g} / \mathrm{ml}$ of ascorbic acid (Sigma Aldrich, St Louis, Missouri) and $15 \mathrm{mM} \quad \mathrm{N}$-2-hydroxyethylenepiperazine-N'-2ethanesulfonic acid (HEPES) buffer 1M (Gibco/Invitrogen). All cultures were maintained at $37^{\circ} \mathrm{C}$ in a humidified $5 \%$ $\mathrm{CO}_{2}$ incubator (Jouan). The culture medium was changed every two to three days. Phase-contrast light microscopy (Olympus, Tokyo, Japan) was used to observe chondrocyte morphology on a daily basis.

Autologous chondrocyte-fibrin construct formation. Articular chondrocytes were cultured in $175 \mathrm{~cm}^{2}$ cultured flasks (Falcon) at a density of $5000 \mathrm{cells} / \mathrm{cm}^{2}$. Once confluenced, the cells were detached using $0.05 \%$ trypsin-EDTA (Gibco/ Invitrogen) and counted with a haemocytometer. Approxi- 
Table I. Modification of the O'Driscoll histological scoring scale ${ }^{41}$ for evaluation of regenerated cartilage and bone according to Frenkel et $\mathrm{al}^{42}$ (reprinted with permission from Elsevier)

\begin{tabular}{|c|c|c|c|}
\hline Category & & & Points \\
\hline \multirow[t]{5}{*}{ I. } & \multirow[t]{5}{*}{ Percentage hyaline articular cartilage: } & 80 to 100 & 8 \\
\hline & & 60 to 79 & 6 \\
\hline & & 40 to 59 & 4 \\
\hline & & 20 to 39 & 2 \\
\hline & & 0 to 19 & 0 \\
\hline \multirow[t]{13}{*}{ II. } & \multicolumn{3}{|l|}{ Structural characteristic } \\
\hline & \multirow{3}{*}{ a. Surface regularity: } & Smooth \& intact & 2 \\
\hline & & Fissures & 1 \\
\hline & & Severe disruption, fibrillation & 0 \\
\hline & \multirow[t]{3}{*}{ b. Structural integrity: } & Normal & 2 \\
\hline & & Slight disruption, including cysts & 1 \\
\hline & & Severe lack of integration & 0 \\
\hline & \multirow[t]{3}{*}{ c. Thickness: } & $100 \%$ of normal adjacent cartilage & 2 \\
\hline & & $50 \%$ to $99 \%$ of normal cartilage or thicker than normal & 1 \\
\hline & & $0 \%$ to $49 \%$ of normal cartilage & 0 \\
\hline & \multirow[t]{3}{*}{ d. Bonding to adjacent cartilage: } & Bonded at both ends of grafts & 2 \\
\hline & & Bonded at one end/partially at both ends & 1 \\
\hline & & Not bonded & 0 \\
\hline \multirow[t]{3}{*}{ III. } & \multirow[t]{3}{*}{ Freedom from cellular changes of degeneration: } & Normal cellularity, no clusters & 2 \\
\hline & & Slight hypocellularity, $<25 \%$ chondrocyte clusters & 1 \\
\hline & & Moderate hypocellularity/hyper, $>25 \%$ clusters & 0 \\
\hline \multirow[t]{4}{*}{ IV. } & \multirow[t]{4}{*}{ Freedom from degenerative changes in adjacent cartilage: } & Normal cellularity, no clusters, normal staining & 3 \\
\hline & & Normal cellularity, mild clusters, moderate staining & 2 \\
\hline & & Mild or moderate hypocellularity, slight staining & 1 \\
\hline & & Severe hypocellularity, poor or no staining & 0 \\
\hline \multirow[t]{3}{*}{ V. } & \multirow[t]{3}{*}{ Reconstitution of subchondral bone: } & Complete reconstitution & 2 \\
\hline & & More than $50 \%$ reconstitution & 1 \\
\hline & & $50 \%$ or less reconstitution & 0 \\
\hline \multirow[t]{3}{*}{ VI. } & \multirow[t]{3}{*}{ Bonding of repair cartilage to de novo subchondral bone } & Complete and uninterrupted & 2 \\
\hline & & $<100 \%$ but $>50 \%$ complete & 1 \\
\hline & & $<50 \%$ complete & 0 \\
\hline \multirow[t]{4}{*}{ VII. } & \multirow[t]{4}{*}{ Safranin O staining } & $>80 \%$ homogeneous positive stain & 2 \\
\hline & & $40 \%$ to $79 \%$ homogeneous positive stain & 1 \\
\hline & & $<40 \%$ homogeneous positive stain & 0 \\
\hline & & Total score (maximum 27 points) & \\
\hline
\end{tabular}

mately 30 million cells $/ \mathrm{ml}$ were incorporated into fresh autologous fibrin. ${ }^{34-36}$ This mixture was then polymerised with $250 \mathrm{mM}$ of calcium chloride $\left(\mathrm{CaCl}_{2}\right)$. Fibrin was used as a temporary scaffold material to support the cells in a three-dimensional (3D) environment. This fresh fibrin was derived from the sheep's own plasma. The plasma was extracted from whole sheep blood collected in sodium citrate tubes by $4800 \mathrm{rpm}$ centrifugation for 5 min, at $4^{\circ} \mathrm{C}$ (Jouan centrifuge). The resulting $3 \mathrm{D}$ gel-like ACFC was maintained for one week in vitro in a complete culture medium prior to the actual implantation. A small ACFC segment was sent for histological assessment.
Implantation of the autologous chondrocyte-fibrin construct. During surgery the left stifle joint was opened. Full-thickness punch hole defects penetrating into the subchondral bone (about $2.5 \mathrm{~mm}$ total depth) were created in the lateral and medial femoral condyles. One defect was implanted with ACFC $(n=12)$ and the other was implanted with either autologous chondrocytes (ACI) $(n=4)$ or autologous fibrin blank construct (AF) (n =6). The untreated defects were left empty $(\mathrm{n}=2)$. Precisely fitting periosteal flaps taken from the proximal medial tibia were applied to cover the defects, with the cambium layer facing the subchondral bone. The flaps were fixed securely by interrupted 6.0 Vicryl sutures 


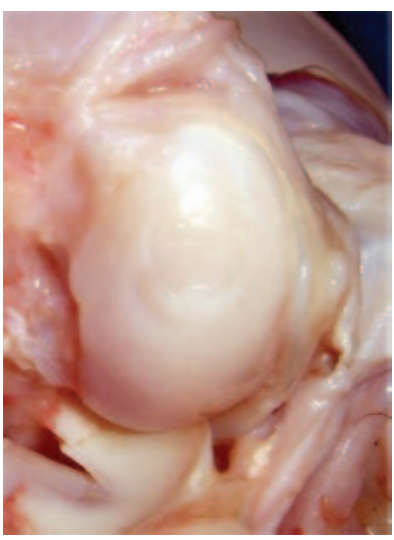

Fig. 1a

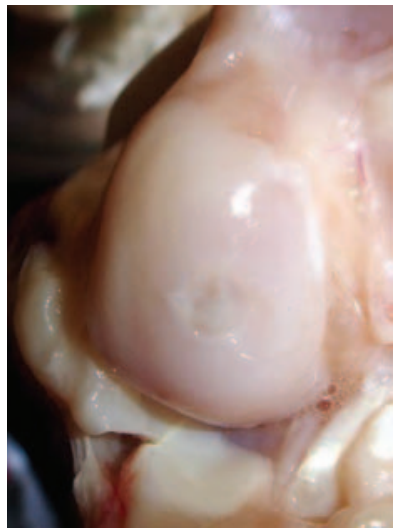

Fig. 1c

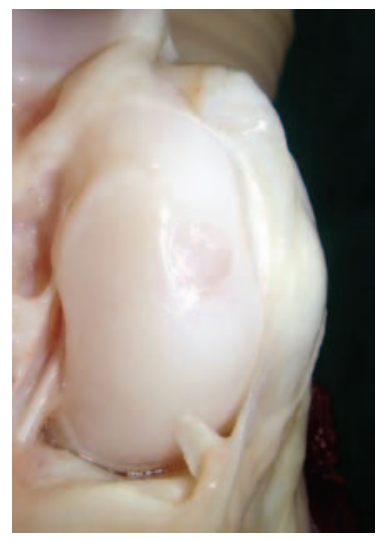

Fig. 1b

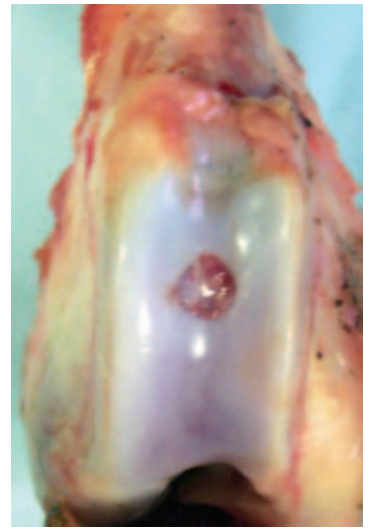

Fig. 1d
Macroscopic view of the repaired osteochondral defects three months after implantation. The colour of a) autologous chondrocyte-fibrin construct-treated defects and b) autologous chondrocyte-treated defects was relatively similar to that of normal adjacent cartilage, whereas the colour of c) the autologous fibrin-treated defects and d) the untreated defects varied from white to tan-purple or red. The texture ranged from irregular to smooth.

(Ethicon, Johnson \& Johnson, Brussels, Belgium). Before the implantation step, autologous fibrin was applied to the surrounding rim of the defects. A watertight seal was confirmed by injecting saline solution under the flap. Cultured chondrocytes were then injected beneath the periosteal flap as described by Brittberg et al. ${ }^{13}$ Before closure, the joint was rinsed thoroughly and the capsule sutured with resorbable stitches, 3.0 Vicryl (Johnson \& Johnson). The total number of transplanted grafts was 22 (12 ACFC, 4 ACI, 6 AF) and two blank defects. The sheep were extensively observed for one week after surgery. They were allowed to move freely without immobilisation and to undertake unrestricted outdoor activities.

Macroscopic assessment. After 12 weeks, the animals were killed by IV injection of $8.0 \mathrm{ml}$ pentobarbital 200 $\mathrm{mg} / \mathrm{ml}$ (Dorminal-20\%, Alfasan, Woerden, Holland) together with $2.0 \mathrm{~g}$ of ketamine (Bioketan, Vetoquinol
Biowet) given intramuscularly. Both stifle joints were harvested, grossly evaluated and photographed. The gross appearance of repaired tissues was assessed in accordance with the International Cartilage Repair Society (ICRS) classification. ${ }^{40}$ Biologically acceptable repairs were defined as having smooth, firm repair tissue that filled the defect and appeared to be attached to the adjacent cartilage. The ICRS classification score for overall repair assessment as: grade I normal, 12 points; grade II, nearly normal, 11 to 8 points; grade III abnormal, 7 to 4 points and grade IV, severely abnormal; 3 to 1 points was used. Samples were excised en bloc for histological analysis and divided into two sections, of which one was fixed in $10 \%$ buffered formalin for 24 hours for histological analysis, and another fixed in $4 \%$ glutaraldehyde for six hours for scanning electron microscopic analysis.

Histological evaluation. After $10 \%$ buffered formalin fixation, all in vivo specimens were decalcified in $8.0 \%$ hydrochloric acid $(\mathrm{HCl})$ solution saturated with ethylenediamine tetraacetic acid (EDTA) for ten days. In vitro ACFC and in vivo samples were dehydrated in a graded series of ethanol, embedded in paraffin, and sectioned at a thickness of $5.0 \propto \mathrm{m}$ using a Leica microtome (Leica Microsystems, Wetziar, Germany). Haematoxylin and eosin (H\&E) and safranin $\mathrm{O}$ staining was performed on all sections and visualised using a light microscope (Olympus). All sections were assessed according to the modified O'Driscoll, Keeley and Salter ${ }^{41}$ histological-histochemical grading scale. ${ }^{10,42}$ Perfect healing of the defect was characterised by 27 points (Table I). In this study, scores were determined using histological sections and included both the defect area and the adjacent native cartilage.

Scanning electron microscopy. After primary fixation in $4 \%$ glutaraldehyde, all samples were soaked with $0.1 \mathrm{M}$ sodium cacodylate for 10 minutes and washed three times. Samples were transferred into $1 \%$ osmium tetraoxide for two hours as post fixation. All were then washed again with $0.1 \mathrm{M}$ sodium cacodylate. Samples were dehydrated in a graded series of acetone, placed into the critical point dryer (CPD Baltec-030) for 30 minutes, mounted on to a stub, sputtered with gold coating in Sputter Coater Polaron E-5100 scanning electron microscopy SEM Coating Unit and viewed under a JEOL-JSM 6400 scanning electron microscope unit.

Statistical analysis. Because of the nature of the semiquantitative scale scoring system the Wilcoxon MannWhitney test was used to compare the two populations. This test is used to test the null hypothesis that two populations have identical distribution functions against the alternative hypothesis that the two distribution functions differ only with respect to location (median). Small significance values $(\mathrm{p}<0.05)$ indicate that the two groups have different locations. 
Table II. Scores according to the International Cartilage Repair Society ${ }^{40}$ classification* $^{*}$

\begin{tabular}{ll}
\hline Treatment & Three months after implantation \\
\hline Autologous chondrocyte-fibrin construct & 10.0 \\
Autologous chondrocyte implantation & 9.5 \\
Autologous fibrin blank & 8.5 \\
Empty (control) & 8.0 \\
* Values are given as the median score (in points) for each category. Biologically \\
acceptable repairs were defined as a smooth, firm repair tissue that filled the defect \\
and appeared to be attached to the adjacent cartilage. There was no significant differ- \\
ence ( $p$ > 0.05 ) between the total median score for ACFC versus ACI ( $p=0.07$ ), but the \\
ACFC score was significantly better than AF blank ( $p=0.04$ ) and empty control \\
( $p=0.003$ ). However, the total median score for each treatment correspond to a nearly \\
normal appearance. Overall repair assessment: \\
Grade I & 12 points \\
Grade II & 11 to 8 points \\
Grade III & 7 to 4 points \\
Grade IV & 3 to 1 points
\end{tabular}

\section{Results}

All sheep tolerated both unilateral arthrotomies well. They were able to stand on all four limbs immediately after surgery, and could walk without limping after five to seven days. No infections or complications occurred throughout post-transplantation monitoring.

Macroscopic observations of cartilage defects. After 12 weeks there was no evidence of infection, arthrofibrosis or fibrinous overgrowth of the joint surface. There was no osteophyte formation, altered synovium or degenerative arthritis. The surface of the repaired tissues showed a nearly normal contour and the texture ranged from irregular to smooth. The colour of ACFC-treated defects (Fig. 1a) and ACI-treated defects (Fig. 1b) was mainly white, which was similar to the adjacent cartilage. However, the colour of fibrin blank (AF)-treated defects (Fig. 1c) and the untreated defects (Fig. 1d) varied from white to tan-purple or red. Visually, the transplanted graft integrated well with the adjacent cartilage. All transplants could be identified easily, as they showed relatively clear demarcation between transplanted graft and adjacent cartilage except for the ACFC-treated defects, which had good integration with the surrounding cartilage. Using the ICRS classification all defects treated with ACFC, ACI and AF exhibited median scores of 10.0, 9.5 and 8.5, respectively, which corresponded to a nearly normal appearance (Table II). Statistically, there was no significant difference between the ACFC and ACI scores.

Standard histological evaluation. After one week, stabilised in vitro ACFC specimens had a homogenous distribution of immature chondrocytes. Underdeveloped proteoglycanrich protein in the in vitro construct was evidenced by the negative safranin $\mathrm{O}$ staining, similar to previously reported results. ${ }^{19}$

After 12 weeks, significant cartilage formation with high cell density was noted extending into the ACFC (Fig. 2) and ACI-treated defects (Fig. 3). Cells were located within a typical lacunar space surrounded by metachromatic matrix. The cells resembled well-differentiated chondrocytes embedded within cartilaginous matrix sim- ilar to native tissue (Fig. 4). Subchondral bone formation was evidenced in both ACFC and ACI specimens, although the repaired tissues did not shown columnar alignment like that of the native tissue. Histological sections were stained positive with safranin $\mathrm{O}$ (orange-red colour) to indicate the presence of accumulated proteoglycan-rich matrix. The transplanted grafts revealed both lateral and basal chondral and osseous integration with the adjacent cartilage in most cases: ACFC (Fig. 2) and ACI (Fig. 3). A hypocellular area could be clearly identified at the demarcation line. However, no sign of nonunion, surface fibrillation or fissure was seen. On the other hand, the defect treated with AF blank exhibited minimal cartilage ingrowth (Fig. 5). The untreated defect (empty control) was fully occupied with disorganised fibrous tissue (Fig. 6) which did not restore a continuous articular surface with the adjacent cartilage. Also, ACFC and ACI significantly enhanced cartilage repair with respective median histological scores of 21.5 and 16.0, compared with AF blank-treated defects (7.5) and untreated defects (0) (Table III).

Scanning electron microscopy examination. Scanning electron micrography of ACFC-treated defects (Fig. 7a) showed a spherical chondrocyte structure attached to the dense extra-cellular matrix and was similar to the native cartilage in Figure 7b. However, in the sagittal cross-section of ACI-treated defects (Fig. 7c) the cell was structurally more elongated and was attached to a thin loose collagen fibril network compared with native cartilage (Fig. 7b) and ACFC (Fig. 7a). Interestingly, the presence of a chondrocyte-secreting matrix was evident in both ACFC- and ACI-treated defects, indicated by the presence of cells with closely packed microvilli on the surface (Fig. 7d). In some fields the presence of healthy proliferative cells in the repaired tissue was indicated by round chondrocytes and flattened, branched pseudopodia-like structures in the lacunar space (Fig. 7e). However, the presence of sponge-like pattern cells in a few fields indicated damage to the chondrocyte structure (Fig. 7f). At 


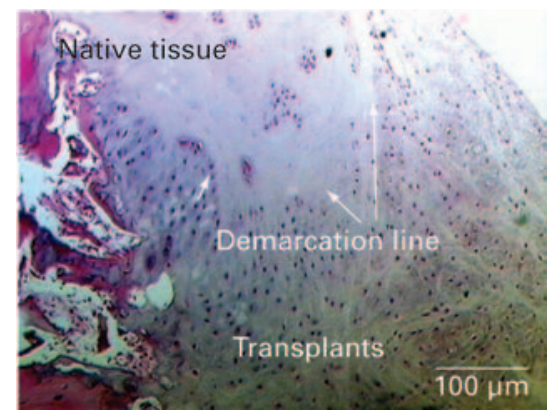

Fig. 2a

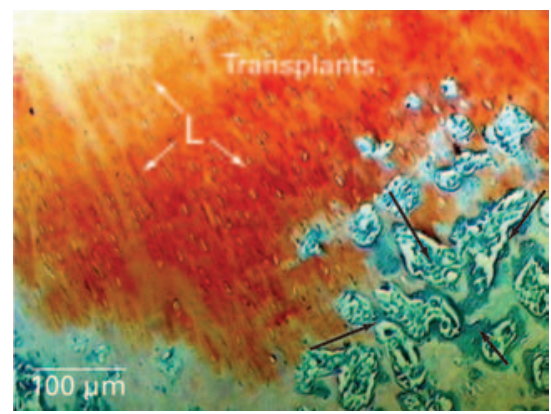

Fig. 2d

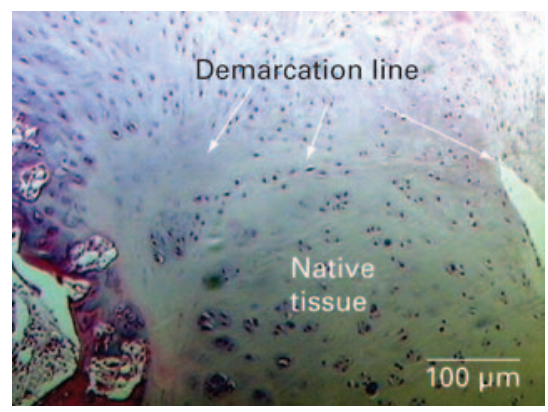

Fig. 3a

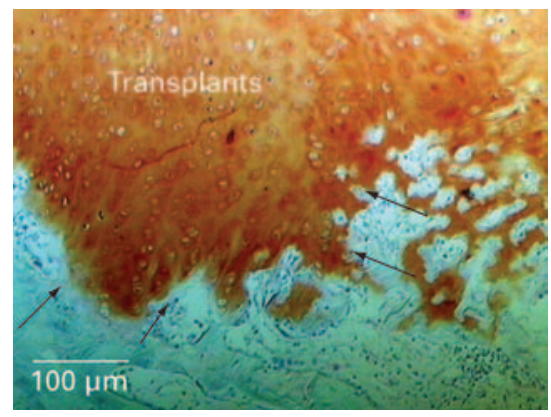

Fig. 3d

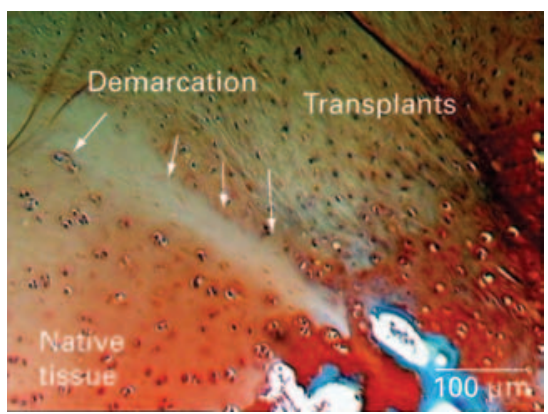

Fig. $2 b$

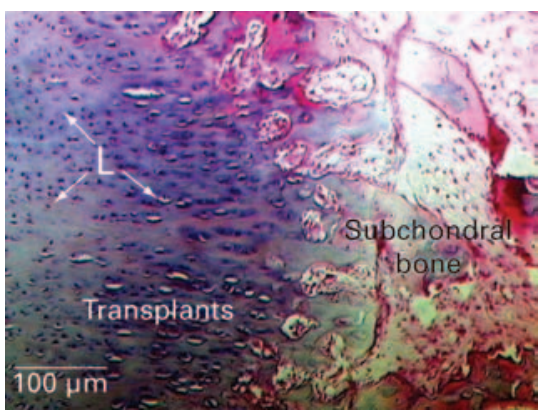

Fig. 2c

Histological examination of the ACFC (a-d) using haematoxylin and eosin and safranin $\mathrm{O}$ staining. After 12 weeks there was significant cartilage formation with high cell density (L) extending into the defect (a-d original magnification $\times 50$ ). Note the formation of new bone (black arrows). The histological sections stained positive with safranin $\mathrm{O}$, indicating the presence of proteoglycan cartilage-rich matrix. The connection between the grafts and adjacent tissues revealed excellent chondral and osseous integration (white arrows). There was no evidence of nonunion.

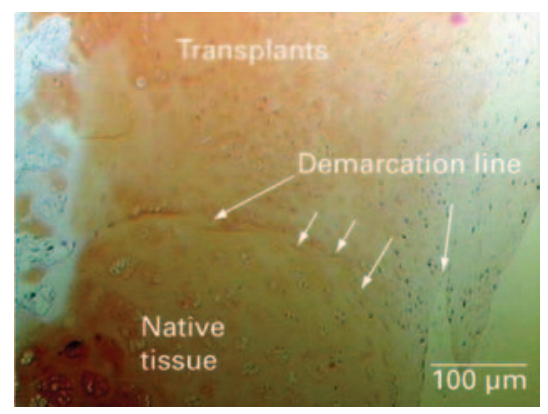

Fig. $3 b$

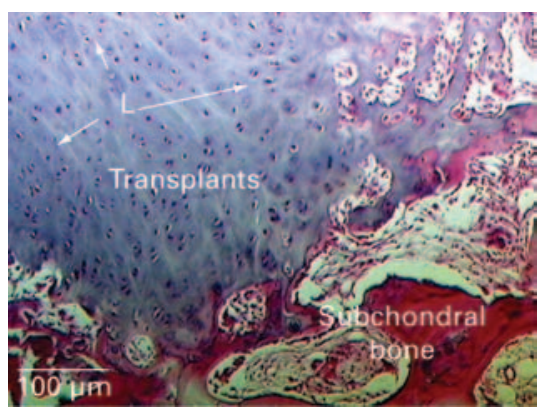

Fig. 3c

Histological examination of the $\mathrm{ACl}$ (a-d) using haematoxylin and eosin and safranin $\mathrm{O}$ staining After 12 weeks there was significant cartilage formation with high cell density (L) extending into the defect (a-d original magnification $\times 50$ ). Note the formation of new bone (black arrows). The histological sections stained positive with safranin 0 , indicating the presence of proteoglycan cartilage-rich matrix. The connection between the grafts and adjacent tissues revealed excellent chondral and osseous integration (white arrows). There was no evidence of nonunion.

low magnification, SEM micrographs of ACFC (Fig. 7g) and ACI (Fig. 7h) revealed excellent chondro-osseous and chondrochondral integration.

\section{Discussion}

Regenerative medicine using tissue-engineering techniques has been given much attention in orthopaedic fields, espe- 


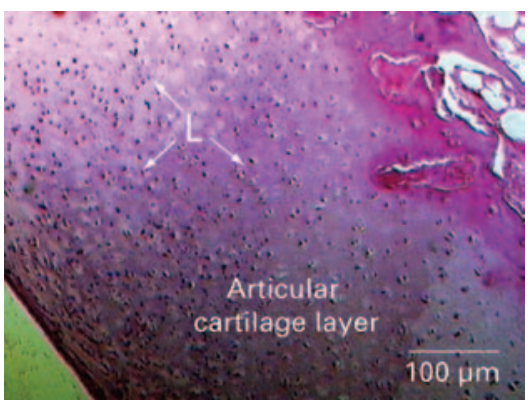

Fig. $4 a$

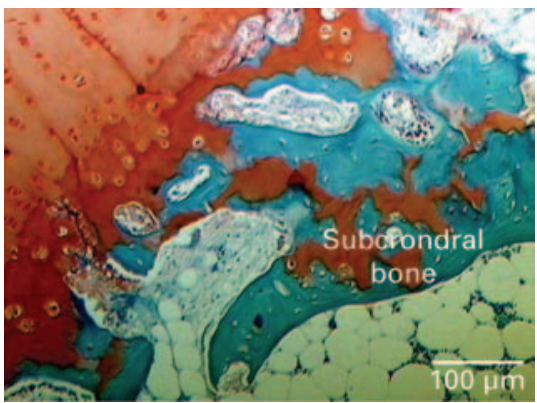

Fig. 4d

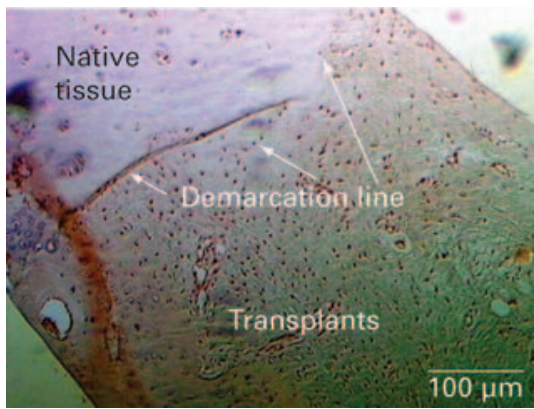

Fig. 5 a

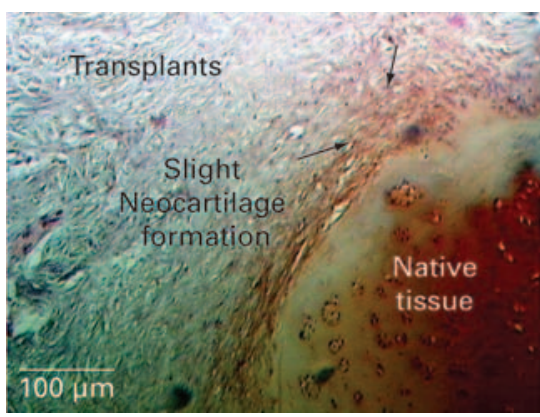

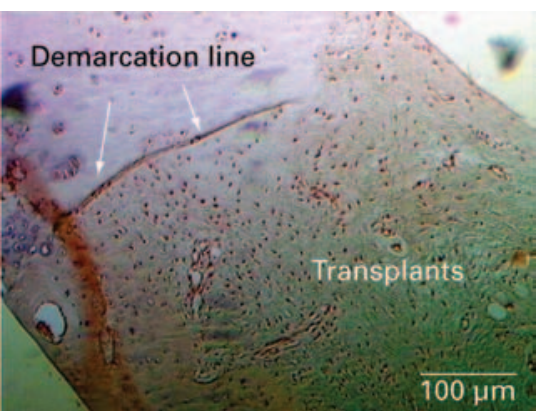

Fig. $5 b$

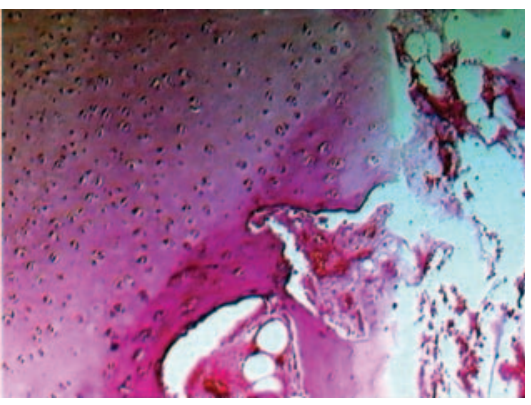

Fig. 4c

Histological examination of the normal cartilage (a-d) using haematoxylin and eosin and safranin O staining ( $L$, chondrocytes in lacunae).

Histological examination of the AF blank-treated defect (a-d) using haematoxylin and eosin and safranin 0 staining. In contrast to Figures 2 to 4 , the defect treated with autologous fibrin blank only exhibited minimal chondral ingrowths (marked with arrows) (a-d original magnification $\times 50$ ).

Fig. $5 d$

cially in the management of cartilage repair. ${ }^{3,37}$ To date, there is very little information available for sheep in the NIH GenBank. ${ }^{43}$ Unfortunately, there is no information quoted for articular cartilage in the nucleotide databases.

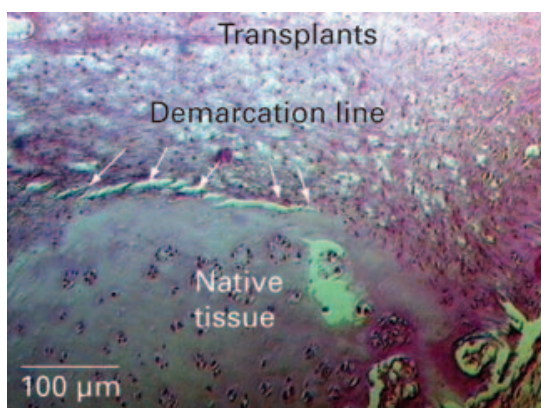

Fig. $5 \mathrm{c}$
Therefore we evaluated the repaired tissues thoroughly using gross inspection and histological examination.

The three-month results demonstrated that chondrocytes trapped within the fibrin network were able to differentiate 


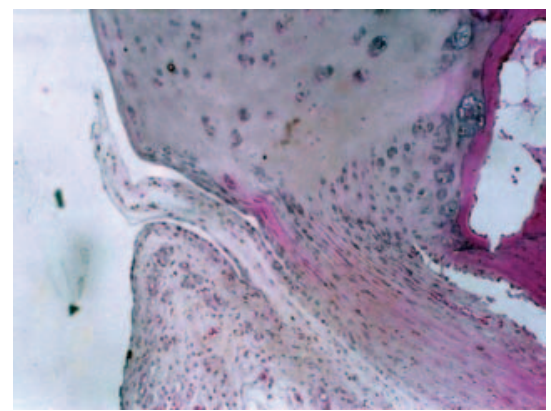

Fig. $6 a$

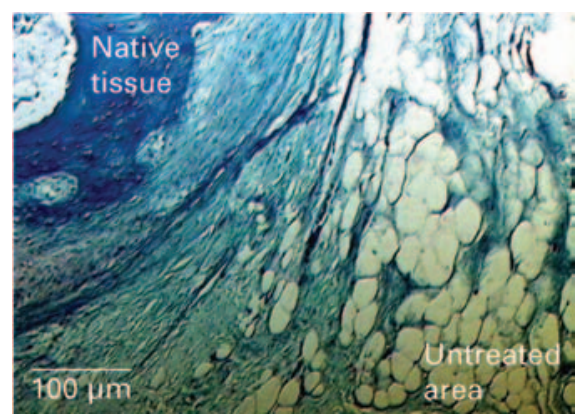

Fig. 6d

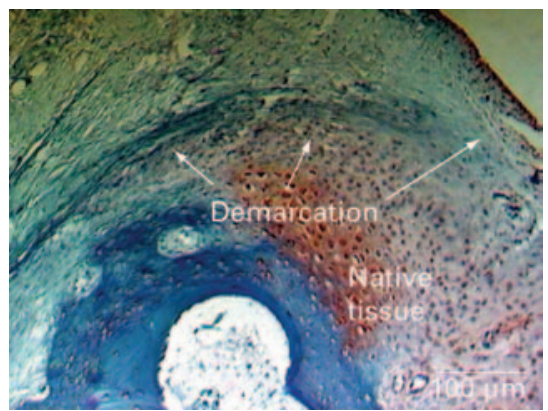

Fig. 6b

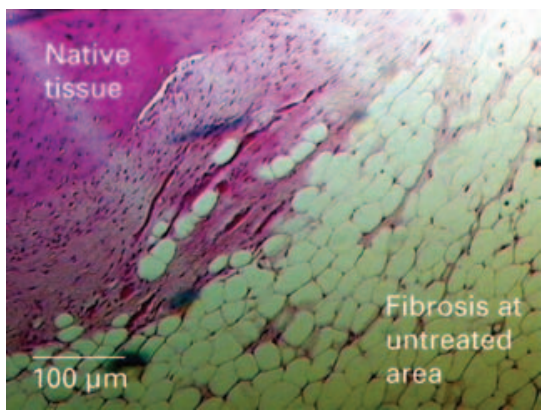

Fig. 6c

Histological examination of the untreated defect (empty control, a-d) using haematoxylin and eosin and safranin $\mathrm{O}$ staining. Generalised fibrosis deposits were present throughout these empty controls (a-d original magnification $\times 50$ ).

Table III. Scores according to the modified histological grading scale $\mathrm{e}^{42}$

\begin{tabular}{|c|c|c|c|c|c|c|c|c|c|c|c|}
\hline \multirow[b]{3}{*}{ Treatment } & \multicolumn{10}{|c|}{ Category $^{*}$} & \multirow{3}{*}{$\begin{array}{l}\text { Total median } \\
\text { score }\end{array}$} \\
\hline & \multirow[t]{2}{*}{ 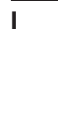 } & \multicolumn{4}{|l|}{ II } & \multirow[t]{2}{*}{ III } & \multirow[t]{2}{*}{ IV } & \multirow[t]{2}{*}{ V } & \multirow[t]{2}{*}{ VI } & \multirow[t]{2}{*}{ VII } & \\
\hline & & A & B & C & D & & & & & & \\
\hline ACFC & 6.0 & 1.0 & 2.0 & 1.0 & 2.0 & 2.0 & 2.5 & 1.0 & 2.0 & 2.0 & 21.5 \\
\hline $\mathrm{ACl}$ & 5.0 & 1.0 & 2.0 & 0 & 2.0 & 1.0 & 2.0 & 1.0 & 1.0 & 1.0 & 16.0 \\
\hline AF blank & 2.0 & 1.0 & 1.5 & 0 & 2.0 & 0 & 1.0 & 0 & 0 & 0 & 7.5 \\
\hline Empty (control) & 0 & 0 & 0 & 0 & 0 & 0 & 0 & 0 & 0 & 0 & 0 \\
\hline
\end{tabular}

and produce good-quality cartilage-like tissue. This supports the hypothesis that ACFC implantation would influence hyaline-like cartilage regeneration. Additionally, this study confirms the findings of Ruszymah and coworkers. ${ }^{19-28,36-38}$ In parallel, several researchers have successfully fabricated injectable tissue-engineered cartilage using fibrin glue matrix seeded with chondrocytes from calf $^{30}$ and swine. ${ }^{31,32}$ Another animal study by Shao et $\mathrm{al},{ }^{4}$ using allogeneic bone marrow mesenchymal stem cells (BMSCs) seeded on to fibrin glue matrix and mPCL, also demonstrated that the cell was able to differentiate and produce cartilage-like neo-tissue after three months in a rabbit model. However, some of the transplants had deteriorated by six months, which they considered to be a result of degradation of the fibrin glue matrix.
Macroscopically, ACFC- and ACI-treated groups had a similar colour to the adjacent cartilage. However, ACFC demonstrated a smoother surface than ACI and AF blank. Surface erosion and fissure formation were rarely observed. There was a variation in thickness of repaired tissues in all cases, as some of the area appeared slightly thicker than the adjacent cartilage. Ito et $\mathrm{al}^{6}{ }^{6}$ who studied cartilage repair using rabbit articular chondrocytes and an atelocollagen sponge/PLLA mesh composite, reported that the repaired tissue had a smoother surface and the thickness of the tissue was about twice that of normal cartilage after 12 weeks. No tissue hypertrophy was observed in this experiment, although there are several studies in humans reporting that approximately $10 \%$ to $15 \%{ }^{44}$ or up to $40 \%^{15}$ of 


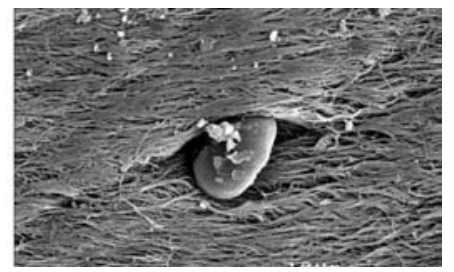

Fig. 7a

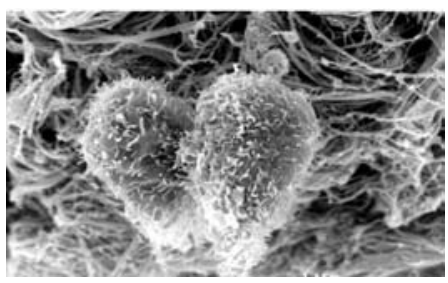

Fig. 7d

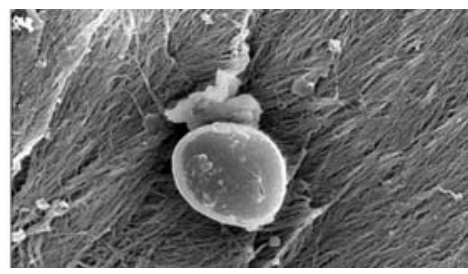

Fig. $7 b$

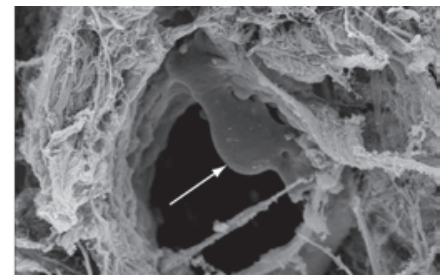

Fig. 7e

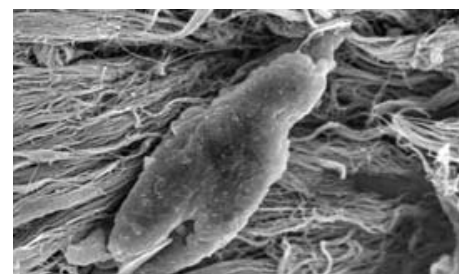

Fig. 7c

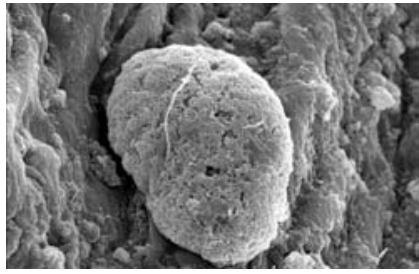

Fig. $7 f$

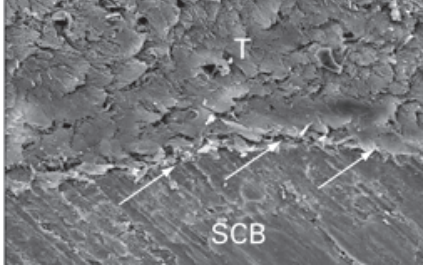

Fig. $7 g$

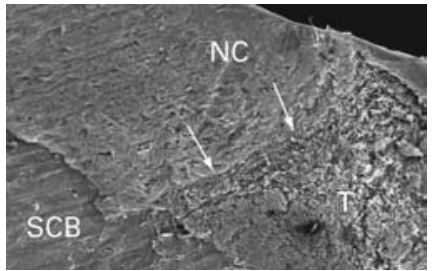

Fig. $7 \mathrm{~h}$

Scanning electron micrographs. a) A sagittal cross-section of autologous chondrocyte-fibrin construct-treated defect. The dense extracellular matrices were similar to the native cartilage (b), with spherical chondrocyte located within lacunar spaces $(\times 3000)$, b) the native cartilage showed dense extracellular matrices with spherical chondrocyte attached to the surface (original magnification $\times 3000$ ), c) micrograph of the autologous chondrocytes implantation-treated defect. The cell is more elongated and attached to a network of thin/loose collagen fibrils $(\times 3000)$; chondrocyte-secreting matrix is evidenced by the presence of cells with closely packed microvilli on their free membrane surface, d) $\times 5000$, e) $\times 700$ shows the chondrocytes (arrow) in the lacunar space. The presence of cells with a sponge-like pattern during SEM observation indicates that the ultrastructure of chondrocytes was destroyed, f) $\times 2500$; low-magnification views of sagittal cross-sections of the defect areas treated with autologous chondrocyte-fibrin construct, g) $\times 330$ and autologous chondrocyte implantation, and $\mathrm{h}) \times 150$. The repaired tissues integrated well with the adjacent cartilage (integration line marked with arrow) (SCB, subchondral bone; NC, native cartilage; $T$, implant).

patients had hypertrophy after receiving periosteal flap and chondrocyte applications.

The histomorphological features and matrix composition of ACFC-treated defects seem superior to those of ACI and AF blank. Most of the repaired tissues demonstrated minimal fibrocartilage formation in the middle of the defect, where the healing process takes place most slowly. However, the fibrocartilage formation in this study should be regarded as newly organised repair tissue, because the invasion of BMSCs from the subchondral bone may produce fibrous tissue during osteochondral repair. ${ }^{7}$ In a few cases a mild inflammatory reaction was seen at the defect site, and in one case neovascularisation was observed in the middle of the ACI-treated defect. Healing of the underlying subchondral bone is essential, as it offers support for the overlying cartilage regeneration. The formation of subchondral bone was evident in the ACFC and ACI groups compared with AF blank, although we have implanted single-phase transplants regardless of bone formation at the defect sites. Thus, the infiltration of host BMSCs into a fibrin matrix network should be considered to facilitate subchondral bone formation. The integration of the repaired tissues with the normal adjacent cartilage was excellent, but the margin of the original defect can be easily identified. However, because of excellent bonding between transplants and the native cartilage, Niederaurer et $\mathrm{al}^{7}$ had difficulty in identifying the original margin in their study of osteochondral repair in a goat model using multiphase implants consisting of poly $(\mathrm{d}, \mathrm{l})$ lactide co-glycolide as a base scaffold material.

Scanning electron microscopic analysis on the morphology of repaired tissues showed the organisation of chondrocytes and matrices in both ACFC- and ACItreated defects, which was relatively similar to that of 
native articular cartilage. The ability of chondrocytes to retain a spherical configuration suggests a well-differentiated phenotype. Chondrocytes characteristically had no direct cell-cell contact and were separated by a sparse territorial matrix, as normally seen in native tissue. The chondrocytes that were retained in the collagenous lacunar space are dedicated to resynthesising and reorganising the associated matrix. In some cases, production of a pericellular matrix by the chondrocyte was clearly visible. Matrix composition was increased from a few collagenous fibrils to form fully established fibril networks that were morphologically indistinguishable from those of normal tissue.

In this study, expansion of articular chondrocyte culture and the formation of an autologous 3D chondrocyte-fibrin construct has allowed us to bypass some methodological concerns regarding the treatment currently in clinical use. This pragmatic concern includes the shortage of donor tissue, donor site morbidity for autograft transplantation, immune rejection, and pathogen transfer with allograft transplantation, delayed inflammatory response in most resorbable scaffolds, and the limited service life or toxicity of arthroplasties. However, in the search for ways to re-establish normal joint function using minimally-invasive surgery, we performed arthrotomy on the animal joints in this study. This technique was employed because of the size of the sheep stifle joints. However, in the future we anticipate the possibility of an arthroscopic procedure on a larger joint such as the human knee.

These findings support the hypothesis that ACFC promotes early chondrogenesis by inducing hyaline-like cartilage regeneration at 12 weeks post-surgery. From the macroscopic and histological findings we concluded that cartilage healing in ACFC was superior to that in controls. Moreover, chondro-osseous and chondrochondral integration between implanted ACFC with surrounding tissues was excellent. ACFC implantation could improve the outcome following conventional ACI. The next step is to evaluate the repaired tissue biomechanically and biochemically over a longer period.

This study was made possible by IRPA grants 06-02-02-0063-EA304 and 06-0202-003 BTK/ER/022 from the Ministry of Science, Technology and Innovation (MOSTI) Malaysia, Faculty of Medicine, National University Malaysia (UKM), National University Malaysia Hospital and Faculty of Veterinary Medicine, Universiti Putra Malaysia. The authors are grateful for the technical assistance of $\mathrm{N}$. Adelina Ahmad Noruddin, S. Sulaiman, M. Adha P. Rameli, Dr M. Y. Loqman, Dr I. S. Norhayati and Dr G. K. Mohd Azam Khan for assisting in sheep management. We thank Mr S. Mohamed and Mrs F. Akmal, the SEM expertise of Microscopy Imaging and Nanoscience Unit, Institute of Bioscience, Universiti Putra Malaysia, for their assistance.

No benefits in any form have been received or will be received from a commercial party related directly or indirectly tot he subject of this article.

\section{References}

1. Hunter W. Of the structure, disease of articulating cartilages: 1743. Clin Orthop 1995:317:3-6.

2. Mankin HJ, Mow VC, Buckwalter JA. Articular cartilage repair and osteoarthritis. In: Orthopaedic basic science: biology and biomechanics of the musculoskeletal system. American Academy of Orthopaedic Surgeons. Second ed. 2000:471-88.
3. Redman SN, Oldfield SF, Archer CW. Current strategies for articulat cartilage repair. Eur Cell Mater 2005;9:23-32.

4. Shao XX, Hutmacher DW, Ho ST, Goh JCH, Lee EH. Evaluation of a hybrid scaffold/cell construct repair of high load bearing osteochondral defects in rabbits. Biomaterials 2006;27:1071-80.

5. Willers C, Chen J, Wood D, Xu J, Zheng MH. Autologous chondrocytes implantation with collagen bioscaffold for the treatment of osteochondral defects in rabbits. Tis Eng 2005;11:1065-76.

6. Ito $\mathbf{Y}$, Ochi $\mathbf{M}$, Adachi $\mathbf{N}$, et al. Repair of osteochondral defect with tissue engineered chondral plug in rabbit model. Arthroscopy 2005;21:1155-63.

7. Niederaurer GG, Slivka MA, Leatherbury NC, et al. Evaluation of multiphase implants for repair of focal osteochondral defects in goats. Biomaterials 2000;21:2561-74

8. van Susante JLC, Buma P, Schuman L, et al. Resurfacing potential of heterologous chondrocytes suspended in fibrin glue in large full-thickness defects of femoral articular cartilage: an experimental study in the goat. Biomaterials 1999;20:1167-75

9. Kose GT, Korkusuz F, Ozkul A, et al. Tissue engineered cartilage on collagen and PHBV matrices. Biomaterials 2005;26:5187-97.

10. Mainil-Varlet P, Rieser F, Grogan S, et al. Articular cartilage repair using a tissueengineered cartilage-like implant: an animal study. Osteoarthritis Cartilage 2001;9(Suppl A):6-15.

11. Tanaka H, Mizokami H, Shigi E, et al. Effects of basix fibroblast growth factor on the repair of large osteochondral defects of articular cartilage in rabbits: doseresponse effects and long term outcomes. Tis Eng 2004;10:633-41.

12. Tibesku CO, Szuwart T, Kleffner TO, et al. Hyaline cartilage degenerates after autologous osteochondral transplantation. J Orthop Res 2004;22:1210-14.

13. Brittberg $\mathbf{M}$, Lindahl A, Nilsson $\mathbf{A}$, et al. Treatment of deep cartilage defects in the knee with autologous chondrocyte transplantation. N Engl J Med 1994;331:889-95.

14. Brittberg M, Talheden T, Sjögren-Jansson E, Lindahl A, Peterson L. Autologous chondrocytes used for articular cartilage repair: an update. Clin Orthop 2001;391(Suppl):337-48.

15. Peterson L, Minas T, Brittberg M, et al. Two to nine year outcome after autologous chondrocyte transplantation of the knee. Clin Orthop 2000;374:212-34.

16. Browne $J E$, Anderson $A F$, Arciero $R$, et al. Clinical outcome of autologous chondrocyte implantation at 5 years in US subjects. Clin Orthop 2005;436:237-45.

17. Horas U, Pelinkovic D, Herr G, Aigner T, Schnettler R. Autologous chondrocyte implantation versus osteochondral cylinder transplantation in cartilage repair of the joint: a prospective, comparative trial. J Bone Joint Surg [Am] 2003;85-A:185-92.

18. Marijnissen WJCM, van Osch GJVM, Aigner J, Verwoerd-Verhoeff HL, Verhaar JAN. Tissue-eingineered cartilage using serially passaged articular chondrocytes: chondrocytes in alginate, combined in vivo with synthetic (E210) or biologic biodegradable (DBM). Biomaterials 2000;21:571-80.

19. Ruszymah BHI, Chua KH, Munirah S, Nur Adelina AN, Aminuddin BS. Tissue engineered cartilage with different human chondrocyte sources: articular, auricular and nasal septum. Med J of Islamic Acad Sci2005;15:5-12.

20. Munirah S, Aminuddin BS, Samsudin OC, et al. The re-expression of collagen type II, aggrecan and sox 9 in tissue engineered human articular cartilage. Tis Eng Reg Med 2005;2:347-55.

21. Munirah S, Aminuddin BS, Chua KH, et al. Tissue-engineered human articular cartilage demonstrates intense immunopositivity for collagen type II. J Biosciences 2006;17:9-18.

22. Munirah S, Aminuddin BS, Chua KH, et al. Tissue engineered human articular neocartilage using serial expanded chondrocytes. Med J Malaysia 2004;59/Suppl E):9-10.

23. Chua KH, Aminuddin BS, Fuzina NH, Ruszymah BHI. Insulin-transferrin-selenium prevent human chondrocyte dedifferentiation and promote the formation of high quality tissue engineered human hyaline cartilage. Eur Cell Mater 2005;9:58-67.

24. Chua KH, Aminuddin BS, Fuzina NH, Ruszymah BHI. Interaction between insulin-like growth factor-1 with other growth factors in serum depleted culture medium for human cartilage engineering. Med J Malaysia 2004;59(Suppl B):7-8.

25. Chua KH, Aminuddin BS, Fuzina NH, Ruszymah BHI. Human serum provided additional values in growth factors supplemented medium for human chondrocytes monolayer expansion and engineered cartilage construction. Med J Malaysia 2004;59(Suppl B):194-5.

26. Nur Adelina AN, Aminuddin BS, Munirah S, et al. Phenotypic expression of collagen type II and collagen type I gene in monolayer culture of human auricular chondrocytes. Med J Malaysia 2004;59(Suppl B):188-9. 
27. Farah Wahida I, Aminuddin BS, Munirah S, et al. Gene expression characteristic in human auricular cartilage tissue engineering. Med J Malaysia 2004;59/Suppl B):190-1.

28. Saim L, Aminuddin BS, Munirah S, et al. Formation of tissue-engineered human auricular cartilage via tissue engineering technique for future use in ear surgery. Med J Malaysia 2004;59(Suppl B):192-3.

29. Ruszymah BHI. Autologous human fibrin as the biomaterial for tissue engineering Med J Malaysia 2004;59(Suppl B):30-1.

30. Sim CD, Butler PE, Cao YL, et al. Tissue engineered neocartilage using plasma derived polymer substrates and chondrocytes. Plast Recons Surg 1998;101:1580-5.

31. Xu JW, Zaporojan V, Peretti GM, et al. Injectable tissue engineered cartilage with different chondrocytes sources. Plast Recons Surg 2004;113:1361-71.

32. Silverman RP, Passaretti D, Huang W, Randolph MA, Yaremchuk MJ. Injectable tissue engineered cartilage using a fibrin glue polymer. Plast Recons Surg 1999;103:1809-18.

33. Fussenegger $\mathbf{M}$, Meinhart $\mathbf{J}$, Hobling $\mathbf{W}$, et al. Stabilized autologous fibrinchondrocyte construct cartilage repair in-vivo. Ann Plast Surg 2003;51:493-8.

34. Samsudin OC, Aminuddin BS, Munirah S, et al. In vitro development of autologous tissue engineered human articular neocartilage for orthopaedic surgery. Med J Malaysia 2004;59(Suppl B):15-16.

35. Badrul AHMY, Aminuddin BS, Sharaf I, et al. The effects of autologous human serum on the growth of tissue engineered human articular cartilage. Med J Malaysia 2004;59(Suppl B):11-12.
36. Azmi B, Aminuddin BS, Sharaf I, et al. The significance of using pooled human serum in human articular cartilage tissue engineering. Med J Malaysia 2004:59(Suppl B):13-14.

37. Ruszymah BHI, Samsudin OC, Munirah S, et al. Repair of articular cartilage after implantation with autologous engineered cartilage made using autologous fibrin as the biomaterial: a fully autologous system [abstract]. World Congress on Tissue Engineering \& Regenerative Medicine. 2006

38. Munirah S, Samsudin OC, Loqman MY, et al. The potential of autologous cartilage implantation in ovine focal cartilage defects: a new option for articular cartilage restoration [abstract]. Tis Eng 2006;12:1014.

39. No authors listed. Universiti Kebangsaan Malaysia Animal Ethics committee (UKMAEC). http://www.medic.ukm.my/laru/UKMAEC-web-ushm2.htm (date last accessed 15 August 2007).

40. Brittberg M, Aglietti P, Gambardella R, et al. ICRS Cartilage Injury Evaluation Package: 200. http://www.cartilage.org/files/ICRS_evaluation.pdf (date last accessed 4 April 2006).

41. O'Driscoll SW, Keeley FW, Salter RB. Durability regenerated articular cartilage produced by free autogenous periosteal grafts in major full-thickness defects in joints surfaces under the influence of continuous passive motion: a follow-up report at one year. J Bone Joint Surg [Am] 1988;70-A:595-606.

42. Frenkel SR, Bradica G, Brekke JH, et al. Regeneration of articular cartilage: an evaluation of osteochondral defect repair in the rabbit using multiphasic implants. Osteoarthritis Cartilage 2005;13:798-807.

43. No authors listed. NIH GenBank. http://www.ncbi.nlm.gov/ (date last accessed 9 August 2007).

44. Minas T, Peterson L. Advanced techniques in autologous chondrocyte transplantation. Clin Sports Med 1999:18:13-44. 